\title{
Abstracts of the meeting of the Association of Clinical Cytogeneticists held on 4 to 6 July 1990 at the University of Manchester, Owens Park
}

\author{
Chromosome band 1p13 is most \\ frequently involved in structural \\ rearrangements in human breast \\ tumours \\ E L D MITCHELL, \\ M F SANTIBANEZ-KOREF \\ Cancer Genetics, Lab 2, Paterson \\ Institute for Cancer Research, Christie \\ Hospital, Manchester.
}

Cytogenetic data of 14 breast tumours analysed in this laboratory are presented. A number of chromosome regions, $16 q, 1 p, 17 p, 6 q, 3 q$, and more specifically bands $1 \mathrm{pl} 3$ and $16 \mathrm{q} 22$, were frequently involved in structural changes in these tumours. These observations together with published cytogenetic data were analysed to determine which regions of the genome are preferentially involved in structural chromosome aberrations. Data from 113 tumours showed that 229 of 302 chromosome bands are involved, the most frequent being band 1 p13 in $20 \%$ of tumours. Other bands frequently represented were $1 \mathrm{pl1}$, 1p22, 1q21, lq23, and 3p11. These breakpoints may indicate the location of genes involved in the aetiology of human breast cancer.

Sample characteristics and outcome of cytogenetic investigation in acute lymphoblastic leukaemia J M HAWKINS, L M SECKER-WALKER Cytogenetics Unit, Department of Academic Haematology, The Royal Free Hospital Medical School, London.

Data on 200 samples received from 190 adults with acute lymphoblastic leukaemia were analysed with respect to sample type (bone marrow or peripheral blood), sample cell count (concentration and total count), time in transit (days), and year of analysis. Each sample was assessed for the number and quality of metaphases and the presence or absence of a clone. Sample type, concentration of cells, time in transit, and year of analysis all significantly affected the number and quality of metaphases; sample type and time in transit had a statistically significant effect on the presence of a clone. Defining a complete success as more than 10 cells fully analysed, the chance of a completely successful cytogenetic analysis in adult ALL was shown to be as low as $10 \%$ with a suboptimal sample (peripheral blood, low cell concentration) and as high as $70 \%$ with an optimal one (bone marrow, adequate cell concentration, one day or less in transit). Cytogenetic findings on six posterior
uveal melanomas

K SISLEY, J RENNIE, D COTTAM,

A POTTER, C POTTER, R REES

Section of Tumour Biology and

Immunology, Department of

Experimental and Clinical Microbiology, Royal Hallamshire Hospital, Sheffield.

Six posterior uveal melanomas were karyotyped after short term culture. One showed a normal chromosome complement and the remaining five had limited chromosome changes. Involvement of chromosomes 1 and 6 was noted in two and four cases respectively and three ciliary body tumours showed both monosomy 3 and $i(8 q)$.

Autologous bone marrow transplantation in CML patients using long term bone marrow cultures C J HARRISON, J CHANG

Regional Cytogenetics Service for Oncology, Paterson Institute for Cancer Research, Christie Hospital, Manchester.

Intermittent high dose hydroxyurea is used for preservation of $\mathrm{Ph}$ negative haemopoietic progenitor cells in CML.
Marrow cells from patients who are in stable chronic phase CML are assessed in the long term bone marrow culture system (LTBMC) for conversion to $\mathrm{Ph}$ negative haemopoiesis. These investigations are totally dependent on cytogenetics for the monitoring of conversion from $\mathrm{Ph}$ positive to $\mathrm{Ph}$ negative status in the patients' marrow and cultures. In patients where conversion is found, an autologous bone marrow transplant, using the purging technique of LTBMC is carried out.

Prenatal diagnosis of an apparently unbalanced familial translocation with normal phenotype

J C K BARBER, N M GREGSON, R LAMBERT

Wessex Regional Genetics Laboratory, General Hospital, Fisherton Street, Salisbury.

Amniocentesis on a 37 year old woman (G4P3) referred for age showed an apparently unbalanced karyotype interpreted as $45, \mathrm{XX},-9,-22,+\operatorname{der}(9)$ $\mathrm{t}(9 ; 22)(9 \mathrm{pter} \rightarrow$ 9q34::22q11 $\rightarrow$ 22qter).

The expected $\operatorname{der}(22)(22 \mathrm{pter} \rightarrow 22 \mathrm{q} 11::$ $9 \mathrm{q} 34 \longrightarrow 9 \mathrm{qter})$ reciprocal product was not found. To establish promptly whether or not this absence might be of clinical significance, blood was requested from both parents as well as their children. Both the mother and two of the three children carried the same apparently unbalanced translocation. The absence of any phenotypic effect in these carriers suggests that the breakpoints in this case may be genuinely juxtacentromeric on chromosome 22 and telomeric on chromosome 9. This family further illustrates the importance of checking familial karyotypes before decisions affecting a pregnancy are taken, even with apparently unbalanced structural rearrangements. 
De novo ring chromosome 3: a new case

M McKINLEY*, A COLLEY $\dagger$, P SINCLAIRt, T ANDREWS' *Department of Medical Genetics, Churchill Hospital, Oxford; tDepartment of Medical Genetics, St Mary's Hospital, Manchester.

Cytogenetic examination of cultured lymphocytes was requested on an 18 year old girl with short stature and dysmorphic features. G banded preparations showed the majority of cells $(84 \%)$ to have a $46, \mathrm{XX}, \mathrm{r}(3)(\mathrm{p} 26 \mathrm{q} 29)$ karyotype. The minority of cells showed other karyotypes: monosomy 3 $(8 \%) ; r(3), r(3)(2 \%)$; dic $r(3)(2 \%)$; and various products of ring breakage (4\%). Both parents had apparently normal karyotypes. On clinical examination the female had proportionate short stature, microcephaly, and brachydactyly. This phenotype includes a triangular face, upward slanting palpebral fissures, and micrognathia. The authors are only aware of three other published cases with similar cytogenetic breakpoints. The clinical features of the published cases are similar to those found in the present case.

\section{Apparently homozygous pericentric inversion of chromosome 4 in an infertile man \\ E MALTBY \\ Centre for Human Genetics, Langhill, Manchester Road, Sheffield.}

A 26 year old male referred for chromosome analysis because of azoospermia was found to have a large pericentric inversion of chromosome 4 (p14q25) in an apparently homozygous state. It was considered that this might be relevant to the patient's problem if either pairing problems at meiosis presented or if this represented a case of uniparental disomy. Meiotic and molecular studies have indicated that this rare and interesting finding is probably coincidental to the clinical referral reason.

\author{
Microdeletions and balanced \\ rearrangements associated with \\ specific clinical disorders \\ I BURVILL-HOLMES*, R T HOWELL*, \\ C GARRETT†, S HARRIS*, \\ J O'LOUGHLIN* \\ * $S$ W Regional Cytogenetics Centre, \\ Southmead Hospital, Bristol; \\ tDepartment of Child Health, \\ Royal Devon and Exeter Hospital, \\ Exeter, Devon.
}

A variety of clinical disorders have been mapped to specific loci through their association with breakpoints in apparently balanced rearrangement, or with small chromosomal deletions. The following rare examples are presented. (1) Two cases of blepharophimosis associated with abnormalities of 3q23, one a small interstitial deletion, the other a balanced translocation. (2) Greig's cephalopolysyndactyly with an interstitial deletion of $7 \mathrm{p} 13$. All previous reports of chromosome abnormalities in this syndrome have involved apparently balanced rearrangements. (3) Lissencephaly, familiar as a feature of Miller-Dieker syndrome with a deletion of $17 \mathrm{pl3}$, in a baby with a de novo direct interarm insertion of chromosome 17.

\section{Familial microdeletion of} chromosome 2 without apparent phenotypic effect

R LAMBERT, M N COLLINSON

Wessex Regional Genetics Laboratory, General Hospital, Fisherton Street, Salisbury SP2 7SX.

We report a family in which several members were found to carry a small deletion of chromosome 2, $\operatorname{del}(2)(\mathrm{p} 12.2 \mathrm{p} 13)$, with no apparent phenotypic effect. The proband was a 21 year old female referred for amniocentesis because of a previous trisomy 18 livebirth. The amniocytes showed a pericentric inversion of chromosome 4 and parental blood samples were requested to determine its origin. The father was shown as the carrier of the inverted 4 but the mother was found to have a small deletion of the short arm of chromosome 2 . High resolution analysis has failed to show the presence of the missing material elsewhere in the karyotype. We compare this deletion with four similar published cases.

Why do S phase inhibitors produce elongated chromosomes at metaphase of mitosis?

M CROCKER, H SHERIDAN

Department of Medical Genetics, Churchill Hospital, Oxford.

$S$ phase inhibitors, such as excess thymidine, methotrexate, and fluorodeoxyuridine, are widely used in cytogenetic laboratories to produce extended metaphase chromosomes. However, the mechanism of action is poorly understood. It clearly has to do with the inhibitory action of these chemicals on DNA synthesis and it has been suggested that synchronisation of cultures is an important factor. This presentation describes the results of an investigation which emphasises the hangover effects of inhibition. The experiments look. for delay effects carried through to mitosis and provide some clues as to why elongated chromosomes are produced. The results suggest that neither synchronisation or block/release effects are responsible for elongation but that a delay in completing $S$ phase may be implicated.

An in vivo mouse model for the neuropathological changes characteristic of Alzheimer's disease J J WATERS*, S B DUNNETT $\dagger$, C M WISCHIK $\ddagger$, S J RICHARDS\ ${ }^{*}$ Regional Cytogenetics Laboratory, East Birmingham Hospital, Birmingham; tDepartment of Experimental Psychology, University of Cambridge; $¥ M R C$ Laboratory of Molecular Biology; \$MRC Molecular Genetics Unit, Hills Road, Cambridge.

Subjects with Down's syndrome develop neuropathological changes characteristic of Alzheimer's disease (AD) in middle life. Markers in the obligate Down's region of human chromosome 21 map to chromosome 16 in mouse. We are interested in determining whether overexpression of genes on chromosome 16 in the trisomic mouse would produce similar pathological changes to those observed in human AD. The Ts16 aneuploidy is lethal during embryogenesis but by performing neural transplants of Ts16 embryos into normal adults of the same strain we have shown the development of the pathological features of $\mathrm{AD}$ in these cortical trisomic tissues.

Chromosomal damage in $\gamma$ irradiated lymphocytes trom Alzheimer patients S TOBI*, D LLOYD $\dagger$, J MOQUET $\dagger$, A EDWARDS†, $\mathbf{R}$ ITZHAKI* *Molecular Neurobiology Laboratory, Department of Optometry, UMIST; †National Radiological Protection Board, Chilton, Didcot, OXI1 ORQ.

We have examined chromosome aberrations in lymphocytes, irradiated in $G_{0}$, from patients with senile 
dementia of the Alzheimer type. This is now the commonest form of dementia, affecting some $5 \%$ of persons over the age of 65. An increased chromosomal sensitivity to ionising radiation is well documented in Down's syndrome, a condition where all middle aged sufferers exhibit the neuropathology of AD. Here, lymphocytes from each of five AD patients show an increase in the number of dicentric chromosomes, compared to five age matched, cognitively unimpaired, normal controls. The mean value for $A D$ subjects is about $25 \%$ higher. This increased radiosensitivity points to possible repair defect in $\mathrm{AD}$.

The ICF syndrome: a rare chromosome instability syndrome

R BAULD, E GRACE, N RICHARDS, $P$ M ELLIS

Lothian Area Cytogenetics Laboratory, RHSC, Edinburgh EH9 ILF.

The ICF syndrome is a rare disorder (only seven previous reports) in which combined Immunodeficiency is associated with Centromeric instability and Facial anomalies. Our patient presented aged 2 years with triangular facies, short stature, dry skin, and wiry hair. She is the first child of a consanguineous marriage. We learned subsequently that she has severe combined immunodeficiency. Analysis of the cultured lymphocytes showed a normal female karyotype, with a characteristic 'stretched' appearance of the heterochromatic regions of chromosomes 1 and 16 and less frequently of chromosome 9. In approximately $30 \%$ of cells there was breakage and some somatic pairing in these regions with interchanges between homologous and nonhomologous chromosomes resulting in isochromosomes, deletions, triradials and dramatic 'sunburst' configurations, in which multiple copies, especially of $16 p$ and 16q, could be identified. Metaphases from cultured skin fibroblasts were normal, as were parental karyotypes. A differential diagnosis of Bloom's syndrome was considered although the rearrangements were atypical. The diagnosis of ICF was confirmed when mitomycin $C$ did not increase SCE levels above the normal range found in untreated cultures.

Recurrent ctb (7)(q31) and possible laminin involvement in cutis laxa
J L HURET, D BONNEAU, G GODOT, M LARREGUE

Genetique, Dermatologie, CHU Poitiers, and CNRS 1174, Creteil, France.

A severe form of cutis laxa was found in a newborn female patient. Progressive cardiac insufficiency and emphysema occurred and the patient died at the age of 22 weeks. A karyotype from peripheral blood showed a recurrent chromatid break at $7 q 31.3$. Fibroblasts did not grow. Skin was incubated with antihuman laminin antiserum. The presence of laminin could not be detected in the basement membranes, while control samples were all positive. A defect in the laminin gene could be responsible for this severe form of cutis laxa.

Patterns of fragile $X$ expression and the role of linked DNA probes in diagnosis

J N MACPHERSON, P A JACOBS Wessex Regional Genetics Laboratory, General Hospital, Fisherton Street, Salisbury SP2 7SX.

The level of expression of the Xq27.3 fragile site in human cells is not a reliable indicator either of the degree of mental retardation in the fragile $X$ syndrome or of the carrier status of a patient. Among Wessex families are found a number of high expressing females showing no obvious mental impairment, while several obligate carriers are fragile $X$ negative. Unusually, one phenotypically normal male exhibits a high level of fragile $X$ expression. The progress of the mutation through families can now be followed independently of $\mathrm{fra}(\mathrm{X})$ expression as a result of the recent isolation of several new DNA probes in close linkage with the fragile $\mathrm{X}$ locus. Cytogenetic and DNA profiles of a selection of families are presented showing the importance of DNA markers in the identification of nonexpressing carriers of both sexes.

Preliminary experience with linked probes in the presymptomatic diagnosis of FAP

M RINDL *, F MACDONALD*,

C McKEOWN†, J HAYDON†, M KEIGHLEY $\ddagger$, M HULTÉN* ${ }^{*}$ DNA Laboratory, Regional Genetic Services; fClinical Genetics, East Birmingham Hospital; †Department of
Surgery, Queen Elizabeth Hospital, Birmingham.

The major feature of familial adenomatous polyposis (FAP) is the many hundreds of polyps within the colon and rectum. At least one of these polyps will become malignant from the second decade of life onwards. The FAP gene has been localised to 5q21.22. By using closely linked probes it is possible to carry out presymptomatic diagnosis. The West Midlands FAP register has been established for two years. It contains 42 families with 51 affected subjects and 107 at risk. In the 11 families tested using DNA probes there are 28 affected subjects of which $18(64 \%)$ are informative. The most informative probes were Pi227 13/18 $(72 \%)$ and $Y N 5.4811 / 18(61 \%)$. The 11 families tested also contained 21 persons at risk. In 16, DNA results could be used to reduce the risk. The most informative probe was again $\mathrm{Pi227}$, but it also has the highest recombination risk. Problems occurred in the use of these probes because of pedigree structure, accurate diagnosis of the disease, and because heterogeneity has not been ruled out. Pedigrees will be illustrated to highlight these problems.

Determination of the parental origin of $\mathrm{X}$;autosome translocations by M27 $\beta$ methylation analysis

D ROBINSON*, Y BOYD†, M COLLINSON*, P JACOBS ${ }^{*}$

*Wessex Regional Genetics Laboratory, General Hospital, Fisherton Street, Salisbury SP2 7SX; $+M R C$

Radiobiology Unit, Chilton, Didcot, Oxon OXII ORD.

The parental origins of four de novo balanced $X$;autosome translocations were determined. Three translocations were in females with DMD with a breakpoint in the $\mathrm{Xp} 21$ region and the fourth in a female with an Xq13.1 breakpoint. Previous studies on the origin of such translocations have relied on cytogenetic studies and RFLP analysis of cell hybrids. We have used a simpler method based on the differential methylation of active and inactive $\mathrm{X}$ chromosomes. In balanced $X$;autosome translocations the normal $X$ is inactive in virtually every cell. The probe $M 27 \beta$ recognises a region of $\mathrm{Xp} 21$ which is heterozygous in $90 \%$ of females using the enzyme MspI. Furthermore M27 $\beta$ is undermethylated on inactive $\mathrm{X}$ chromo- 
somes. Therefore Southern blot analysis of parallel $M s p I$ and $H p a I I$ digests of DNA from probands and their parents shows the parental origin of the methylated, active, and therefore translocated X chromosome. All four translocations studied were paternal in origin. A further five $\mathbf{X}$;autosome translocation females with DMD have been documented elsewhere and the translocations found to be paternal in origin in all five.
Recent developments in the CF gene

M SCHWARZ

Paediatric Genetics Unit,

Royal Manchester Children's Hospital, Hospital Road, Manchester M27 1HA.

The major mutation in cystic fibrosis is a three base pair deletion of exon 10 of the cystic fibrosis transmembrane conductance regulator (CFTR) gene, which results in the deletion of a phenylalanine residue at position 508 of the protein product. This mutation ( $\triangle F 508$ ) accounts for some $80 \%$ of $C F$ chromosomes in the north-west of England and is generally associated with a severe form of the disease. The second of a rapidly growing number of mutations to be discovered was also a three base pair deletion in the same exon and, although rare, accounts for some $1.5 \%$ of CF genes in the French population. 